\title{
Vector mode analysis of ring-core fibers: design tools for spatial division multiplexing
}

\author{
Charles Brunet, Bora Ung, Pierre-André Bélanger, Fellow, OSA, Younès Messaddeq, \\ Sophie LaRochelle Senior Member, IEEE, and Leslie A. Rusch, Fellow, IEEE
}

\begin{abstract}
Design tools have existed for decades for standard step-index fibers, with analytical expressions for cutoff conditions as a function of core size, refractive indices and wavelength. We present analytical expressions for cutoff conditions for fibers with a ring-shaped propagation region. We validate our analytical expressions against numerical solutions, as well as via asymptotic analysis yielding the existing solutions for standard step-index fiber. We demonstrate the utility of our solutions for optimizing fibers supporting specific eigenmode behaviors of interest for spatial division multiplexing. In particular we address large mode separation for orbital angular momentum modes and fibers supporting only modes with a single intensity ring.
\end{abstract}

\section{INTRODUCTION}

$\mathbf{T}$ HE surge in interest in space-division multiplexing $(\mathrm{SDM})$ requires new tools for the design of novel optical fibers supporting a variety of mode types. We focus on an annular fiber, or ring-core fiber (RCF), with a step-index ring geometry (Fig. 1) and develop analytical expressions for the eigenmodes supported. Design tools have existed for decades for standard solid-core step-index fiber (SCF), providing analytical expressions for modal cutoff as a function of core size, refractive indices and wavelength. Those expressions are simplified by the use of a single parameter, the normalized frequency $\left(V_{0}\right)$. With RCF, there is now not only one, but two parameters defining the geometry of the core: the ring outer radius, and $\rho$, the ratio of inner radius to outer radius. As a consequence, even when using normalized frequency, cutoff conditions are no longer given by single points.

Cutoff conditions define the boundary between regions supporting specific modes, i.e. a modal map. Analytical relationships are well known for SCF cutoff for both vector and scalar versions of the propagation equations. Solutions for vector versions are valid for arbitrary refractive indices contrast between the core and cladding, and yield mode maps for SCF eigenmodes. This index contrast can be defined by the parameter $n_{0}^{2} \triangleq n_{1}^{2} / n_{2}^{2}$. Under the scalar or weakly guiding assumption (when $n_{0}^{2} \rightarrow 1$ ), the mode maps cover only scalar LP modes.

The authors are with Electrical and Computer Engineering Department, Center for Optics, Photonics and Lasers (COPL), Université Laval, Québec, QC G1V 0A6, Canada (e-mail: rusch@gel.ulaval.ca).

B. Ung is with Department of Electrical Engineering, Ecole de Technologie Superieure, Montréal H3C 1K3, Canada (e-mail: Bora.Ung@etsmtl.ca).

Pierre-André Bélanger is with Physics Department, Center for Optics, Photonics and Lasers (COPL), Université Laval, Québec, QC G1V 0A6, Canada.
The LP mode maps are the simplest and most familiar, however they cannot be used to predict or target support of other modes of interest, including modes carrying discrete states of orbital angular momentum (OAM). On the other hand, eigenmode maps are the most versatile, as they constitute a complete basis set for all propagating modes (including the LP and the OAM modes). This distinction has tremendous impact on fiber design as well as the demultiplexing reception strategy for SDM. We therefore focus on vector mode analysis to provide analytical eigenmode mode maps for RCF.

Annular fibers are of renewed interest because of their applications in SDM [1] and optical sensing [2]. In particular, this type of fiber appears well suited for transmitting OAM modes [3]-[6], because its refractive index profile closely matches that of the annular intensity profile of OAM beams. RCF is also being investigated for few-mode transmission of LP modes [7]-[9], as RCF can be tailored to minimize differential group delay (DGD).

To the best of our knowledge, the analytical description of modal cutoffs in RCF have been limited to LP modes under the weakly guiding approximation [10]-[12]. In light of the recent advances in high-index contrast waveguides and the emerging interest in OAM-guiding fibers, a fully-vectorial description of RCF is necessary. In this paper, the solution of the modal cutoffs for the vector modes of RCFs is derived without any approximations. A summary of the key SCF and RCF modal mapping and cutoff results is provided in Table I.

SDM design goals for RCF for OAM modes transmission require eigenmodes with separations of their effective indices greater than $10^{-4}$ [3]. Greatest effective index separation is achieved at maximal index contrast, in direct violation of the weakly guiding condition where indices are approximately equal [6]. Therefore LP mode maps are doubly inappropriate: 1) results are inaccurate as OAM fibers target high refractive index contrasts, and 2) modal maps are provided for LP modes only, but not the eigenmodes that constitute the OAM modes. While the LP modal maps are still useful, the knowledge of the underlying effective index separations for the eigenmodes based on our vector analysis may be exploited towards gaining precise control over the number of vector modes that can be supported. This is particulary important when designing fibers for OAM transmission.

In section II we define fiber parameters and start from the modal equations to derive the cutoff conditions for all RCF vector modes. In section III we validate our results by examining three asymptotic versions of our results: 1) when 
TABLE I

COMPARISON BETWEEN SCALAR AND VECTOR MODES IN STANDARD AND ANNULAR FIBER

\begin{tabular}{c}
\hline Standard fiber \\
1D
\end{tabular}

$n_{1}^{2} \rightarrow n_{2}^{2}$ (weakly guiding RCF), 2) when the inner ring diameter shrinks to zero (RCF becomes SCF), and 3) when fiber core diameter is very large, i.e. both $a$ and $b \rightarrow \infty$ (becomes like a planar waveguide). In section IV we discuss application of the results of section II to specific SDM design targets, including number of modes supported, targeting a subset of mode types, and maximizing mode separation. We also present an snapshot of where published OAM fibers and fiber designs fall in a modal map for annular fiber. Readers interested in the fiber design alone could skip details in sections II, III and go directly to section IV.

\section{DERIVATION OF THE CHARACTERISTIC EQUATION FOR VECTOR MODES}

\section{A. Fiber parameters and modal equations}

Annular fiber geometry is illustrated in Fig. 1. It is characterized by the inner and outer radii $a$ and $b$, and by the refractive indices of cladding $\left(n_{2}\right)$ and annular core $\left(n_{1}\right)$, where $n_{1}>n_{2}$. This kind of fiber is also known as ring-core fiber [9] or M-type optical fiber [12].

The modal solutions for RCF are found by solving Maxwell equations for the electric $\vec{E}(\vec{r})$ and magnetic $\vec{H}(\vec{r})$ fields, where $\vec{r}=(r, \phi, z)$ in cylindrical coordinates. The timedependent electric and magnetic fields for a mode propagating in the $z$ direction are given by:

$$
\begin{aligned}
\vec{E}(\vec{r}, t) & =\vec{E}(r, \phi) e^{j(\omega t-\beta z)} \\
\vec{H}(\vec{r}, t) & =\vec{H}(r, \phi) e^{j(\omega t-\beta z)}
\end{aligned}
$$

where $\beta$ is the propagation constant, and $\omega$ is the angular frequency.

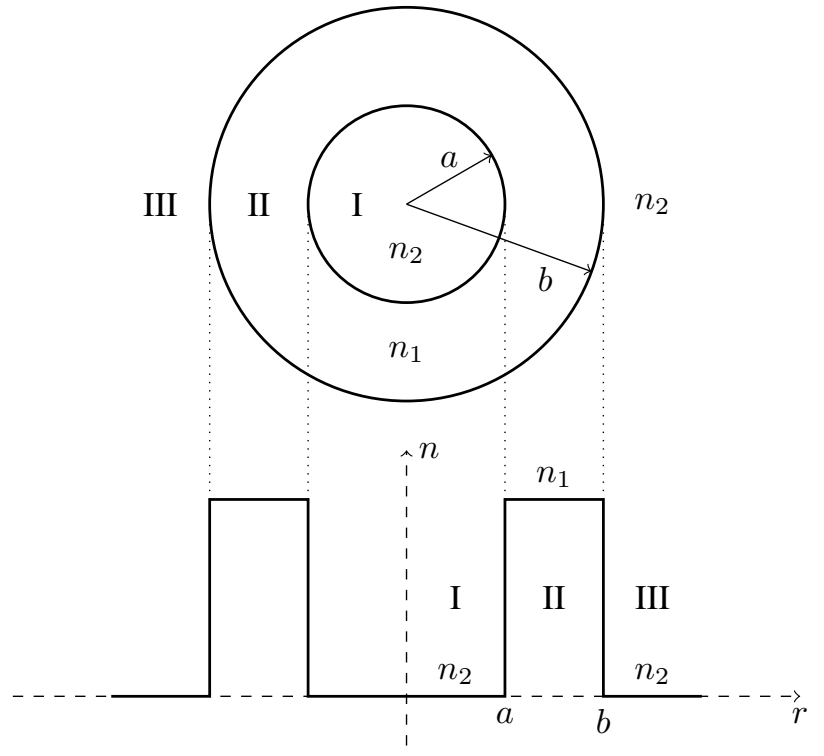

Fig. 1. Annular fiber geometry (top view, and profile).

To solve for this electro-magnetic field, we first specify the longitudinal components $E_{z}$ and $H_{z}$, from which we derive the transverse components. In RCF, the longitudinal fields, obtained from the well-known Helmholtz equations, are of the form:

$$
\begin{aligned}
& E_{z}= \begin{cases}C_{1} I_{\nu}(w r) \cos \left(\nu \phi+\phi_{0}\right) & r \leq a \\
{\left[A_{1} J_{\nu}(u r)+\right.} & a<r \leq b \\
\left.A_{2} N_{\nu}(u r)\right] \cos \left(\nu \phi+\phi_{0}\right) & r>b \\
C_{2} K_{\nu}(w r) \cos \left(\nu \phi+\phi_{0}\right) & r>b\end{cases} \\
& H_{z}= \begin{cases}D_{1} I_{\nu}(w r) \sin \left(\nu \phi+\phi_{0}\right) & r \leq a \\
{\left[B_{1} J_{\nu}(u r)+\right.} & a<r \leq b \\
\left.B_{2} N_{\nu}(u r)\right] \sin \left(\nu \phi+\phi_{0}\right) & r>b \\
D_{2} K_{\nu}(w r) \sin \left(\nu \phi+\phi_{0}\right) & r>b\end{cases}
\end{aligned}
$$

where $\nu \geq 0$, the azimuthal order, is an integer, $\phi_{0}$ is an arbitrary phase angle, $J_{\nu}$ and $N_{\nu}$ are Bessel functions of the first and second kind, and $I_{\nu}$ and $K_{\nu}$ are modified Bessel functions of the first and second kind. Parameters $u$ and $w$ are defined as:

$$
\begin{aligned}
u^{2} & =n_{1}^{2} k_{0}^{2}-\beta^{2} \\
w^{2} & =\beta^{2}-n_{2}^{2} k_{0}^{2}
\end{aligned}
$$

where $k_{0}$ is the wavenumber in the vacuum $(2 \pi / \lambda)$. Propagation constant $\beta$ and the eight constants $\left(C_{1}, A_{1}, A_{2}, C_{2}\right)\left(D_{1}, B_{1}, B_{2}, D_{2}\right) \quad$ are determined by enforcing the continuity of the tangential components $E_{z}, E_{\phi}, H_{z}$, and $H_{\phi}$ at the boundaries, when $r=a$ and $r=b$. These conditions lead to a set of eight homogeneous equations for which the eigenvalues (i.e. the propagation constants $\beta$ ) are found by solving the linear system of equations. The mode index, $\nu$, is an index to multiple solutions to this system of equations, each solution being a mode. 


\section{B. The characteristic equation}

The modal characteristic equation is obtained by calculating the $(8 \times 8)$ determinant of the system of equations. In principle, the analytical solution can be obtained by eliminating the constants $C_{1}, C_{2}$, and $D_{1}, D_{2}$ from the eight equations, thus simplifying the problem to a four equation (5), (6), (7), (8) system.

$$
\begin{aligned}
& \left(\frac{1}{u^{2}}+\frac{1}{w^{2}}\right) \frac{\nu \beta}{a^{2}}\left[A_{1} J_{\nu}(u a)+A_{2} N_{\nu}(u a)\right]= \\
& k_{0}\left\{\left[B_{1} \frac{J_{\nu}^{\prime}(u a)}{u a}+B_{2} \frac{N_{\nu}^{\prime}(u a)}{u a}\right]+\right. \\
& \left.\frac{I_{\nu}^{\prime}(w a)}{(w a) I_{\nu}(w a)}\left[B_{1} J_{\nu}(u a)+B_{2} N_{\nu}(u a)\right]\right\} \\
& \left(\frac{1}{u^{2}}+\frac{1}{w^{2}}\right) \frac{\nu \beta}{a^{2}}\left[B_{1} J_{\nu}(u a)+B_{2} N_{\nu}(u a)\right]= \\
& k_{0}\left\{n_{1}^{2}\left[A_{1} \frac{J_{\nu}^{\prime}(u a)}{u a}+A_{2} \frac{N_{\nu}^{\prime}(u a)}{u a}\right]+\right. \\
& \left.\frac{I_{\nu}^{\prime}(w a)}{(w a) I_{\nu}(w a)} n_{2}^{2}\left[A_{1} J_{\nu}(u a)+A_{2} N_{\nu}(u a)\right]\right\} \\
& \left(\frac{1}{u^{2}}+\frac{1}{w^{2}}\right) \frac{\nu \beta}{b^{2}}\left[A_{1} J_{\nu}(u b)+A_{2} N_{\nu}(u b)\right]= \\
& k_{0}\left\{\left[B_{1} \frac{J_{\nu}^{\prime}(u b)}{u b}+B_{2} \frac{N_{\nu}^{\prime}(u b)}{u b}\right]+\right. \\
& \left.\frac{K_{\nu}^{\prime}(w b)}{(w b) K_{\nu}(w b)}\left[B_{1} J_{\nu}(u b)+B_{2} N_{\nu}(u b)\right]\right\} \\
& \left(\frac{1}{u^{2}}+\frac{1}{w^{2}}\right) \frac{\nu \beta}{b^{2}}\left[B_{1} J_{\nu}(u b)+B_{2} N_{\nu}(u b)\right]= \\
& k_{0}\left\{n_{1}^{2}\left[A_{1} \frac{J_{\nu}^{\prime}(u b)}{u b}+A_{2} \frac{N_{\nu}^{\prime}(u b)}{u b}\right]+\right. \\
& \left.\frac{K_{\nu}^{\prime}(w b)}{(w b) K_{\nu}(w b)} n_{2}^{2}\left[A_{1} J_{\nu}(u b)+A_{2} N_{\nu}(u b)\right]\right\}
\end{aligned}
$$

The characteristic equation can now be retrieved from a $(4 \times 4)$ determinant. However, the corresponding analytical expression would be so long and complicated that it would be of little practical use. Instead, modern personal computers and optimization algorithms directly solve for the modes.

\section{The cutoff frequencies}

The modal cutoff frequencies are obtained for $\beta \rightarrow n_{2} k_{0}$ and $w \rightarrow 0$. In Appendix $\mathrm{A}$ we provide useful recurrence relations and asymptotic limits of various Bessel functions when their argument goes to zero.

The TE and TM mode cutoffs are obtained by setting $\nu=0$, with $A_{1}=A_{2}=0$ for the TE modes, and $B_{1}=B_{2}=0$ for the TM modes. The four continuity equations (5-8) thus reduce to two equations: (5 and 7) for the TE modes, and (6 and 8 ) for the TM set. With the help of the asymptotic relations in Appendix A, we can show that the cutoff frequencies calculated from the $(2 \times 2)$ determinant are given by

$$
J_{0}(u b) N_{2}(u a)-N_{0}(u b) J_{2}(u a)=0
$$

for the $\mathrm{TE}_{0, m}$ modes, and by

$$
\begin{aligned}
J_{0}(u b) N_{2}(u a)-N_{0}(u b) J_{2}(u a)= & \\
& \frac{\left(1-n_{0}^{2}\right)}{n_{0}^{2}}\left[J_{0}(u b) N_{0}(u a)-J_{0}(u a) N_{0}(u b)\right]
\end{aligned}
$$

for the $\mathrm{TM}_{0, m}$ modes, where $n_{0}^{2} \triangleq \frac{n_{1}^{2}}{n_{2}^{2}}$.

When $\nu \neq 0$ and $w \rightarrow 0$, we find that the electric and magnetic longitudinal fields are zero at $r=a$, because of the limits of the $I_{\nu}$ Bessel functions in (2) and (3). Therefore we can write:

$$
\begin{aligned}
& A_{1} J_{\nu}(u a)+A_{2} N_{\nu}(u a)=0 \\
& B_{1} J_{\nu}(u a)+B_{2} N_{\nu}(u a)=0
\end{aligned}
$$

at the cutoff conditions. Equations (5) and (6) are then zero and the continuity relations consist of only (7) and (8), where $A_{2}$ and $B_{2}$ can be eliminated using (11). The determinant of this $(2 \times 2)$ system of equations leads to the following cutoff relation for $\mathrm{HE}$ modes when $\nu \geq 2$ :

$$
\begin{aligned}
& J_{\nu-2}(u b) N_{\nu}(u a)-J_{\nu}(u a) N_{\nu-2}(u b)= \\
& \frac{\left(1-n_{0}^{2}\right)}{\left(1+n_{0}^{2}\right)}\left[J_{\nu}(u b) N_{\nu}(u a)-J_{\nu}(u a) N_{\nu}(u b)\right]
\end{aligned}
$$

As shown in Appendix B, the asymptotic behavior is logarithmic for $\nu=1$, and the resulting cutoff condition becomes

$$
J_{1}(u b) N_{1}(u a)=J_{1}(u a) N_{1}(u b)
$$

The first zero $u a=u b$ is associated with the $\mathrm{HE}_{1,1}$ mode of the annular core fiber, and the following zeros with $\mathrm{HE}_{1, m}$, where $m=2,3, \ldots$.

It is also possible to set the longitudinal electric and magnetic fields at $r=b$ to be zero at cutoff, as it is the case for EH modes in standard fiber. From (2) and (3), this gives

$$
\begin{aligned}
& A_{1} J_{\nu}(u b)+A_{2} N_{\nu}(u b)=0 \\
& B_{1} J_{\nu}(u b)+B_{2} N_{\nu}(u b)=0
\end{aligned}
$$

For $w \rightarrow 0,(7)$ and (8) are zero, and the remaining equations (5) and (6) can be written as a $(2 \times 2)$ system of equations. As shown in Appendix $\mathrm{B}$, the determinant gives the cutoff condition for the $\mathrm{EH}_{\nu, m}$ modes of the annular core fiber:

$$
\begin{aligned}
J_{\nu+2}(u a) & N_{\nu}(u b)-J_{\nu}(u b) N_{\nu+2}(u a)= \\
& \frac{\left(1-n_{0}^{2}\right)}{\left(1+n_{0}^{2}\right)}\left[J_{\nu}(u a) N_{\nu}(u b)-J_{\nu}(u b) N_{\nu}(u a)\right]
\end{aligned}
$$

To conclude this section, our main results are the cutoff equations $(9,10,12,13$, and 15). This completes the analytical study of annular fibers. In the following sections, we will demonstrate the accuracy of these formulas by examining their asymptotic behavior and through numerical validations. 
TABLE II

CUTOFF CONDITIONS FOR STANDARD FIBERS

\begin{tabular}{cl}
\hline Mode & cutoff \\
\hline $\mathrm{TE}_{0, m}, \mathrm{TM}_{0, m}$ & $J_{0}\left(V_{0}\right)=0$ \\
$\mathrm{HE}_{1, m}$ & $J_{1}\left(V_{0}\right)=0$ \\
$\mathrm{HE}_{\nu, m}$ & $J_{\nu-2}\left(V_{0}\right)=\frac{1-n_{0}^{2}}{1+n_{0}^{2}} J_{\nu}\left(V_{0}\right)$ \\
$\mathrm{EH}_{\nu, m}$ & $J_{\nu}\left(V_{0}\right)=0$ \\
\hline
\end{tabular}

\section{VALIDATION}

\section{A. Asymptotic results}

1) $a \rightarrow 0(R C F \rightarrow S C F)$ : A first validation of the cutoff relations for RCF can be performed by the asymptotic analysis when $a \rightarrow 0$. In that case, the center part of the fiber completely collapses and RCF approaches a SCF. Cutoff conditions for SCF are well known, and summarized in Table II.

The asymptotic behavior of Bessel functions $J_{\nu}$ and $N_{\nu}$ when their argument approaches 0 is given in (A.31) and (A.32) in Appendix A. One can easily verify that all the cutoff equations of the RCF $(9,10,12,13$, and 15) collapse as expected to those of the SCF when $a \rightarrow 0$.

2) $n_{1} \rightarrow n_{2}$ (weakly guiding $R C F$ ): Under the weakly guiding approximation $\left(n_{0}^{2} \approx 1\right)$ it can be shown that the cutoff equations reduce to that of the scalar LP modes of the RCF [10]-[12]. In particular, the cutoff equations for the $\mathrm{TE}_{0, m}$ (9) and $\mathrm{HE}_{1, m}$ (13) modes remain the same under the weakly guiding approximation, since they do not contain the $n_{0}^{2}$ term. Moreover, for the $\mathrm{TM}_{0, m}$ modes we can see that the cutoff equation becomes the same as that of the $\mathrm{TE}_{0, m}$ modes, and the same can be said of the $\mathrm{HE}_{2, m}$ modes $(\nu=2)$. Finally, using (12) and (15) we can demonstrate that the $\mathrm{HE}_{\ell-1, m}$ and $\mathrm{EH}_{\ell+1, m}$ modal cutoffs (for $\ell \geq 2$ ) reduce to the degenerate form:

$$
J_{\ell+1}(u a) N_{\ell-1}(u b)=J_{\ell-1}(u b) N_{\ell+1}(u a)
$$

The above analysis (based on the weakly guiding approximation) is in complete agreement with the standard description of LP modes in weakly guiding SCF and RCF. We show in Appendix $C$ that (20) is a simpler but equivalent expression to those found in [10, eq. (8)] and [11, eq. (13)].

3) $a, b \rightarrow \infty$ (planar waveguide): When the ring diameter increases, the fiber becomes highly multimode. In the limit condition where $a, b \rightarrow \infty$, the fiber behaves like a planar waveguide, and the number of guided modes only depends on the ring thickness $d=b-a$. Since planar waveguides only support TE and TM modes, we start the development from (9) and (10).

Substituting (A.35) and (A.36) into (9), we obtain

$$
\begin{aligned}
\cos \left(u b-\frac{\pi}{4}\right) \sin \left(u a-\pi-\frac{\pi}{4}\right)- & \\
\sin \left(u b-\frac{\pi}{4}\right) \cos \left(u a-\pi-\frac{\pi}{4}\right) & = \\
\sin (u(b-a)) & = \\
\sin (u d) & =0
\end{aligned}
$$

Similarly, substituting (A.35) and (A.36) into (10) gives

$$
\begin{aligned}
\sin (u d)+\frac{1-n_{0}^{2}}{n_{0}^{2}} \sin (u d) & = \\
\sin (u d) & =0
\end{aligned}
$$

Therefore, the cutoff condition for both TE and TM modes is the same, as expected for a planar waveguide. The same result could be obtained from the cutoff condition in weakly guiding regime (20). This condition can be expressed as

$$
u d=m \pi
$$

which is the expected cutoff condition for a planar waveguide [13]. A similar demonstration is given in [11].

\section{B. Normalized notation}

In RCF, in contrast to SCF, we have two fiber geometry parameters, namely $a$ and $b$. By defining $\rho \triangleq \frac{a}{b}$, we can normalize our parameters in a similar way to that done in SCF. In this way, the cutoff conditions can be expressed in terms of the normalized frequency $V_{0}$ and the ratio $\rho$ using this equivalent notation:

$$
\begin{aligned}
V_{0} & =u b=k_{0} b \sqrt{n_{1}^{2}-n_{2}^{2}} \\
\rho V_{0} & =u a=k_{0} a \sqrt{n_{1}^{2}-n_{2}^{2}}
\end{aligned}
$$

The cutoff conditions can be written explicitly as a function of these parameters and $n_{0}^{2}$, as seen in Table IV of section IV. We also define the normalized propagation constant as

$$
\widetilde{\beta}=\frac{\beta^{2}-n_{2}^{2} k_{0}^{2}}{n_{1}^{2} k_{0}^{2}-n_{2}^{2} k_{0}^{2}} \approx \frac{n_{\text {eff }}-n_{2}}{n_{1}-n_{2}}
$$

The cutoff frequency occurs for $\beta \rightarrow k_{0} n_{2}$, or $\widetilde{\beta} \rightarrow 0$.

With these definitions we will first validate our analytical results via numerical simulation in the next subsection. These definitions are critical to section IV on design tools for RCF fiber, and will be used extensively there.

\section{Numerical validation}

In this section we will provide a numerical validation of our results for one value of $n_{0}^{2}$, and one value of $\rho$. Fixing these values leaves the normalized frequency $V_{0}$ to determine the normalized propagation constant $\widetilde{\beta}$. We solve for it numerically, using the transfer matrix method [14], to find the vector modes supported. Similar results are obtained in [4] using finite elements method (FEM).

We fixed $n_{0}^{2}$ at 1.042 which corresponds to the case $n_{1}=$ 1.474 and $n_{2}=1.444$. This case has limited index contrast such that the weakly guiding mode is a reasonable assumption. These values are typical of telecommunications silica fiber designs. The ratio $\rho$ was fixed at .25 . Numerical solutions are given in Fig. 2 where each curve is labeled by the vector mode name whose normalized propagation constant is plotted. When $\widetilde{\beta}$ approaches zero we are in the cutoff condition. Hence the asymptotic value of $\widetilde{\beta} \rightarrow 0$ should correspond to our analytical prediction to have validation. 


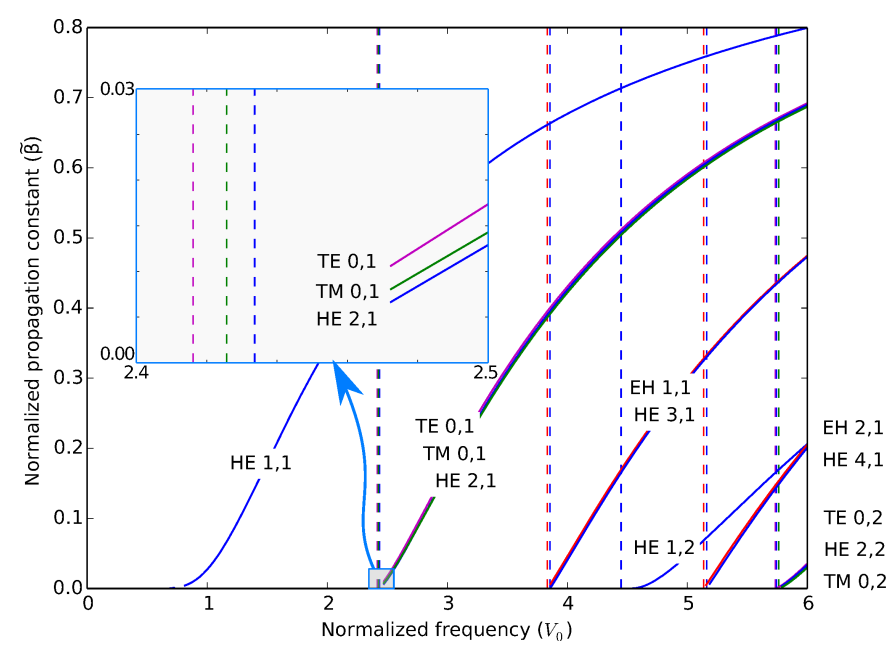

Fig. 2. Normalized propagation constant $\widetilde{\beta}$ as a function of the normalized frequency $V_{0}$, for RCF with $\rho=0.25$ and $n_{0}^{2}=1.042$.

TABLE III

NORMALIZED CUTOFF FREQUENCIES $\left(V_{0}\right)$ AS FUNCTION OF $\rho\left(n_{0}^{2}=1.042\right)$

\begin{tabular}{ccccc}
\hline$\rho$ & 0 & 0.25 & 0.5 & 0.75 \\
\hline $\mathrm{TE}_{0,1}$ & 2.4048 & 2.4161 & 2.5544 & 3.1663 \\
$\mathrm{HE}_{2,1}$ & 2.4221 & 2.4336 & 2.5742 & 3.1943 \\
$\mathrm{TM}_{0,1}$ & 2.4048 & 2.4257 & 2.5822 & 3.2188 \\
$\mathrm{EH}_{1,1}$ & 3.8317 & 3.8330 & 3.9294 & 4.6458 \\
$\mathrm{HE}_{3,1}$ & 3.8533 & 3.8561 & 3.9648 & 4.7123 \\
$\mathrm{HE}_{1,2}$ & 3.8317 & 4.4475 & 6.3932 & 12.6056 \\
$\mathrm{EH}_{2,1}$ & 5.1356 & 5.1359 & 5.1976 & 5.9360 \\
$\mathrm{HE}_{4,1}$ & 5.1597 & 5.1603 & 5.2316 & 6.0074 \\
$\mathrm{TE}_{0,2}$ & 5.5201 & 5.7336 & 7.3236 & 13.3513 \\
$\mathrm{HE}_{2,2}$ & 5.5277 & 5.7418 & 7.3337 & 13.3631 \\
$\mathrm{TM}_{0,2}$ & 5.5201 & 5.7610 & 7.3583 & 13.3822 \\
\hline
\end{tabular}

For each mode we solve equations in Table IV numerically. We present our analytical predictions of $V_{0}$ at $\widetilde{\beta} \rightarrow 0$ in Table III, for $n_{0}^{2}=1.042$ and four values of $\rho$. Values in column two $(\rho=0)$ are the well-known solutions for SCF, and are given as a comparison point.

In Fig. 2, the analytical cutoff values, plotted as vertical dashed lines, correspond to the values in column three of Table III. The numerical solutions for the normalized propagation constant go to zero at the analytical values found for cutoff, as expected.

In the inset, we zoom on the cutoff of the first mode group. We can see how the numerical solutions fall short of reaching the precise cutoff value. We were unable to achieve closer values due to numerical instabilities. Numerical calculation of cutoff frequencies is prone to error as the value of Bessel $K$ function diverges as $w \rightarrow 0$, leading to numerical instabilities. Analytical expressions for cutoff are particularly useful for this reason.

Equation (11) implies that at cutoff the electrical field $E_{z}$ is zero at $r=a$ for HE modes. In a similar way, (14) implies that $E_{z}$ is zero at $r=b$ for $\mathrm{EH}$ modes. Consider the vector modes $\mathrm{HE}_{3,1}$ and $\mathrm{EH}_{1,1}$ for $n_{0}^{2}=1.042$ and $\rho=0.5$ (shaded cutoff frequencies in Table III), which compose $\mathrm{LP}_{2,1}$. We select $V_{0}=4.13$ that is above but near cutoff. We plot in Fig. 3

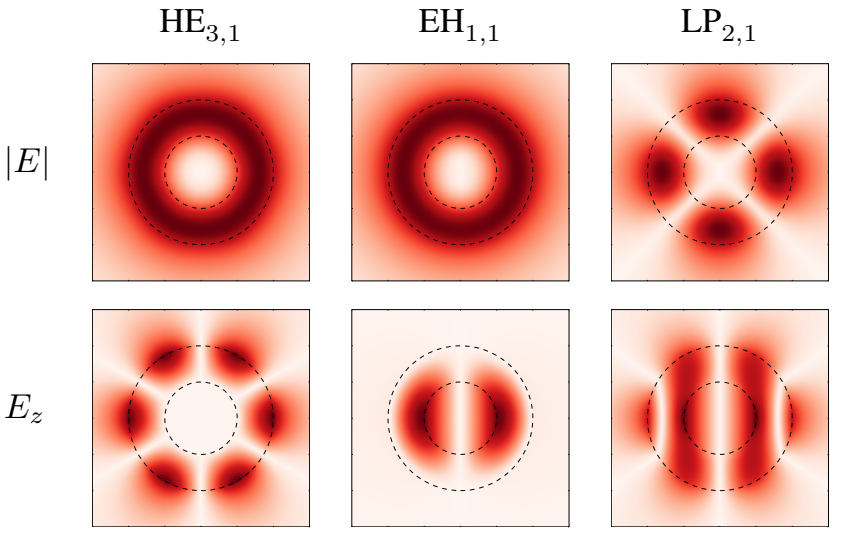

Fig. 3. $E$ field intensity (top) and $E_{z}$ field (bottom), for $\mathrm{HE}_{3,1}, \mathrm{EH}_{1,1}$, and $\mathrm{LP}_{2,1}$ modes, in an annular fiber with parameters: $V_{0}=4.13, \rho=0.5$, and $n_{0}^{2} \stackrel{2,1}{=} 1.042$.

the electric field of the $\mathrm{LP}_{2,1}$ mode group and its constituent eigenmodes. The dashed circles define the boundaries of the annular core for $\rho=0.5$. This plot confirms that near cutoff, $E_{z}$ field of $\mathrm{HE}$ modes is located at the exterior interface of the annular core, while the $E_{z}$ field of EH modes is located at the interior interface of the core.

This completes the validation of cutoff equations for annular fibers. In the next section, we will explore in more detail the use of these analytical expressions for the design of specialty fibers supporting spatial-division multiplexing applications.

\section{Design TOOLS}

In section II, we found analytical expressions for the cutoff conditions in annular fiber. In section III-B, we introduced a normalized parameterization of the fiber using the normalized frequency, $V_{0}$, and $\rho$, the ratio of annular radii. Our results for cutoff conditions are given in Table IV using the new fiber parameterization. Clearly the triplet $V_{0}, \rho$ and $n_{0}^{2}$ completely characterize the cutoff conditions. We will now use these equations to meet specific fiber design goals by exploiting this triplet. For the first two subsections (IV-A and IV-B) we continue with $n_{0}^{2}=1.042$ where the weakly guiding assumption is accurate. Our conclusions will be applicable to LP modes as well as vector modes. In section IV-C we will violate the weakly guiding assumption and target widely separated propagation constants among mode groups. In sections IV-C, IV-D, and IV-E we focus on fibers supporting OAM modes.

\section{A. Modal map}

For each fiber mode, the cutoff condition specifies the functional relationship between $\rho$ and $V_{0}$ for that mode. Those cutoff conditions define boundaries of parameter regions where a specific number of modes are supported (i.e. guided) by the fiber. In a SCF, $\rho=0$, and the number of modes is only determined by intervals of the $V_{0}$ parameters; in this case the modal map only has one dimension. In the case of RCF, the modal map has two dimensions $\left(\rho\right.$ and $\left.V_{0}\right)$. This is illustrated in Fig. 4. The white region on Fig. 4 is the region where the fiber is monomode, thus supports only the $\mathrm{HE}_{1,1}$ mode. Any 
TABLE IV

CUTOFF CONDITIONS FOR ANNULAR FIBERS

\begin{tabular}{cc}
\hline Mode & cutoff \\
\hline $\mathrm{TE}_{0, m}$ & $J_{0}\left(V_{0}\right) N_{2}\left(\rho V_{0}\right)-N_{0}\left(V_{0}\right) J_{2}\left(\rho V_{0}\right)=0$ \\
& $J_{0}\left(V_{0}\right) N_{2}\left(\rho V_{0}\right)-N_{0}\left(V_{0}\right) J_{2}\left(\rho V_{0}\right)=$ \\
$\mathrm{TM}_{0, m}$ & $\frac{\left(1-n_{0}^{2}\right)}{n_{0}^{2}}\left[J_{0}\left(V_{0}\right) N_{0}\left(\rho V_{0}\right)-J_{0}\left(\rho V_{0}\right) N_{0}\left(V_{0}\right)\right]$ \\
& $J_{\nu-2}\left(V_{0}\right) N_{\nu}\left(\rho V_{0}\right)-J_{\nu}\left(\rho V_{0}\right) N_{\nu-2}\left(V_{0}\right)=$ \\
$\mathrm{HE}_{\nu, m}$ & $\frac{\left(1-n_{0}^{2}\right)}{\left(1+n_{0}^{2}\right)}\left[J_{\nu}\left(V_{0}\right) N_{\nu}\left(\rho V_{0}\right)-J_{\nu}\left(\rho V_{0}\right) N_{\nu}\left(V_{0}\right)\right]$ \\
& $\mathrm{HE}_{1, m}\left(V_{0}\right) N_{1}\left(\rho V_{0}\right)=J_{1}\left(\rho V_{0}\right) N_{1}\left(V_{0}\right)$ \\
& $J_{\nu+2}\left(\rho V_{0}\right) N_{\nu}\left(V_{0}\right)-J_{\nu}\left(V_{0}\right) N_{\nu+2}\left(\rho V_{0}\right)=$ \\
$\mathrm{EH}_{\nu, m}$ & $\frac{\left(1-n_{0}^{2}\right)}{\left(1+n_{0}^{2}\right)}\left[J_{\nu}\left(\rho V_{0}\right) N_{\nu}\left(V_{0}\right)-J_{\nu}\left(V_{0}\right) N_{\nu}\left(\rho V_{0}\right)\right]$ \\
\hline
\end{tabular}

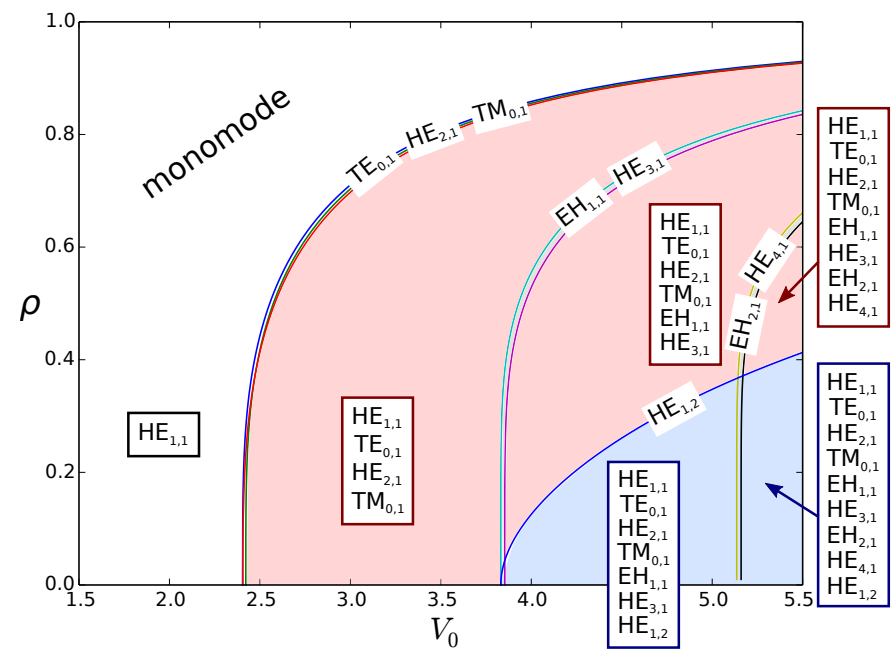

Fig. 4. Modal map defined by the cutoffs of the first order modes as function of $V_{0}$ and $\rho$, with $n_{0}^{2}=1.042$.

combination of $V_{0}$ and $\rho$ falling in that region will yield a monomode fiber. While SCF fibers are monomode only for $V_{0}<2.405$, it is possible to design an RCF with $V_{0}>2.405$ that is still single-mode, assuming $\rho$ is high enough.

The monomode region is delimited by the cutoff of the $\mathrm{TE}_{0,1}$ mode (or $\mathrm{LP}_{1,1}$ mode under the weakly guiding approximation). For $n_{0}^{2}$ near one, the case of Fig. 4, cutoffs of $\mathrm{HE}_{2,1}$ and $\mathrm{TM}_{0,1}$ modes are very close to the cutoff of $\mathrm{TE}_{0,1}$ mode. This is visible on Fig. 4 where there are actually three lines between the monomode region and the region below, but they are too close to be clearly distinguished.

Below the monomode region is a region where the fiber supports the second mode group $\left(\mathrm{TE}_{0,1}, \mathrm{HE}_{2,1}\right.$ and $\mathrm{TM}_{0,1}$ modes, or $\mathrm{LP}_{1,1}$ under the weakly guiding approximation), in addition to the fundamental mode. The next boundary is the cutoff of the third mode group, namely $\mathrm{EH}_{1,1}$ and $\mathrm{HE}_{3,1}$ modes (or $\mathrm{LP}_{2,1}$ ). Fiber in the region between these two boundaries is the simplest case of few-mode fiber (FMF). This includes fibers supporting the first OAM mode $\left(\mathrm{OAM}_{ \pm 1, m}\right)$.
Other regions are defined on a similar way, forming together a modal map. Fig. 2 has vector mode solutions on the boundary curves, and listed in boxes is the set of all modes supported within a region proscribed by the boundaries. The cutoff of the $\mathrm{HE}_{1,2}$ mode forms an interesting boundary. In the regions above that cutoff (red shading), all the supported modes have their $m$ parameter equal to one. Below that limit (blue shading), fiber begins to support modes with $m$ parameter greater than one. We will discuss this in more detail in a later section.

\section{B. Targeting number of supported modes}

To design a monomode fiber, we have to choose parameters to eliminate the first higher-order mode, namely the $\mathrm{TE}_{0,1}$. We write this cutoff condition from (9) as

$$
\frac{J_{0}\left(V_{0}\right)}{N_{0}\left(V_{0}\right)}=\frac{J_{2}\left(\rho V_{0}\right)}{N_{2}\left(\rho V_{0}\right)}
$$

In a similar way, we can find the parameters for an RCF having only a limited number of modes. For example, for a fiber supporting only the $\mathrm{TE}_{0,1}, \mathrm{TM}_{0,1}, \mathrm{HE}_{1,1}, \mathrm{HE}_{2,1}, \mathrm{EH}_{1,1}$, and $\mathrm{HE}_{3,1}$ modes, the modal region limit is approximated by the cutoff of the mode $\mathrm{LP}_{2,1}$ (20):

$$
\frac{J_{1}\left(V_{0}\right)}{N_{1}\left(V_{0}\right)}=\frac{J_{3}\left(\rho V_{0}\right)}{N_{3}\left(\rho V_{0}\right)}
$$

In RCF, the key parameter determining the number of supported modes is $\rho$, the ratio between inner and outer radii of the core. When $\rho$ is very small, the fiber cutoffs are very close to those of a SCF having core radius $b$. When $\rho$ is small to moderate (approximately below 0.4 , as a rule of thumb), the cutoffs of the modes with $m$ parameter equal to one behave like those of SCF, while cutoffs of modes with higher $m$ parameter are at increasing $V_{0}$ for increasing $\rho$. Finally, the characteristics of fibers with large $\rho$ (i.e. very thin ring width) approach those of planar waveguides. For arbitrary $\rho$, fibers with high $V_{0}$ parameter are usually highly multimode.

\section{Targeting mode separation}

The third parameter, $n_{0}^{2}$, dictates the separation between the vector modes. When $n_{0}^{2}$ approaches one, we are under the weakly guiding condition. When $n_{0}^{2}$ increases, the separation between the effective indices of the different modes also increases. Exploitation of LP modes for spatial multiplexing relies on the use of multiple input, multiple output (MIMO) processing. This processing is simplified when the propagation constants of the LP modes are similar. Therefore, when designing a fiber for LP modes, we want $n_{0}^{2}$ to be as close as possible to one, in order to minimize intra-modal dispersion. In the case of OAM modes, MIMO processing may be avoided completely if the inter-modal coupling is minimized by having greatly disparate propagation constants among vector mode groups forming OAM modes. Therefore, for OAM we want $n_{0}^{2}$ as large as possible, violating the weakly guiding assumption. The value of $n_{0}^{2}$ we can reach is usually limited by the properties of the materials used in fiber fabrication. 


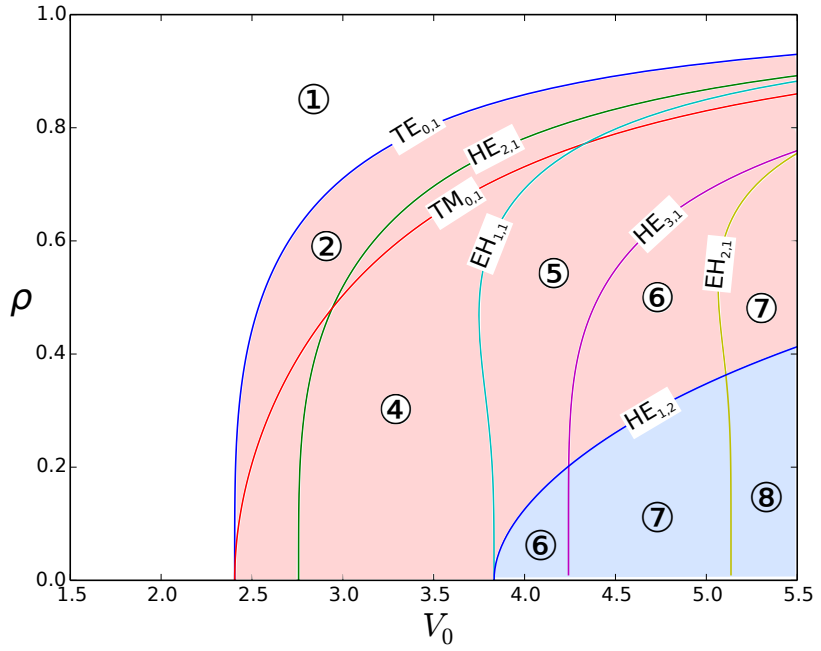

Fig. 5. Modal map defined by the cutoffs of the first order modes as function of $V_{0}$ and $\rho$, with $n_{0}^{2}=2.085$.

For the transmission of OAM modes, we target effective index separation greater than $10^{-4}$, as this value is suggested by the literature [3]. We cannot directly infer the mode separation from the modal map; the modal map only shows the separation at cutoff condition. However, the cutoff separation can still give a rough approximation of the mode separation, as explained in Appendix D.

In the previous two sections we examined scenarios where $n_{0}^{2}$ was near one, typical for the design of FMF for LP spatial multiplexing. Previously developed analytical solutions [10] for RCF could be applied as the weakly guiding assumption is valid. In this subsection we consider the design of OAM modes that require high contrast fibers for greater modal separation. Maximizing the number of supported modes requires the highest contrast possible, motivating the use of a fiber with an air core - that is, air as the innermost guiding medium [5], [6].

Consider the scenario where $n_{1}=1.444$ and $n_{2}=1$ (air), yielding $n_{0}^{2}=2.085$. The modal map for this case is plotted in Fig. 5. Once again, the cutoff curve is labeled with the vector mode it describes; red shading indicates regions with $m=1$, while blue shading indicates $m>1$. The number of vector modes supported in a given region is indicated by a circled number rather than an exhaustive list.

With increased index contrast, we first note that all mode cutoffs are shifted right except $\mathrm{TE}_{0, m}$ and $\mathrm{HE}_{1, m}$. This is as expected from cutoff equations in Table IV, since all equations depend on $n_{0}^{2}$, with the exception of equations for $\mathrm{TE}_{0, m}$ and $\mathrm{HE}_{1, m}$ mode cutoffs. A second important difference is that there now are regions defined inside mode groups. For instance, region marked with (2), i.e. with two modes supported, includes only $\mathrm{HE}_{1,1}$ and $\mathrm{TE}_{0,1}$ modes. While this region also exists when $n_{0}^{2}$ is smaller, it becomes so small that it is of no practical use. The red shaded region supporting five modes, and the blue shaded region supporting six modes are another example, where the $\mathrm{EH}_{1,1}$ mode exists without the $\mathrm{HE}_{3,1}$ mode.

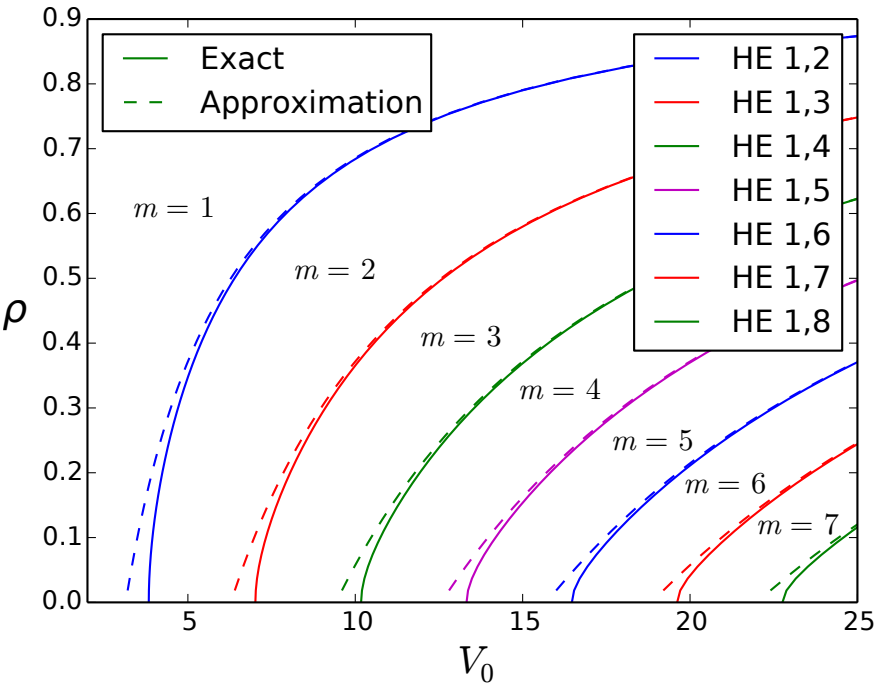

Fig. 6. Delimitations of the different radial orders for annular fiber. Plain lines are the exact solutions, while dashed lines are calculated from the approximation.

\section{Single radial order condition}

We define the radial order as the number of concentric intensity rings in a guided mode, which corresponds to the modal parameter $m$. In the context of LP/OAM modes guiding in a single-ring RCF, it is important to define a single radial order condition which determines the parameter space for which only modes of radial order $m=1$ are excited. An interesting property of the RCF is that as $\rho$ increases (i.e. for thinner core rings), the cutoff of the $\mathrm{HE}_{1,2}$ mode does not follow the cutoff of the $\mathrm{EH}_{1,1}-\mathrm{HE}_{3,1}$ group. Within this single radial order region (red shaded area in Fig. 4), all supported modes are of the first radial order $(m=1)$, meaning that their modal field distribution consists of only one intensity ring which is well-matched to the RCF refractive index profile. Crucially, the latter observation also means that RCFs open up the possibility of supporting a much larger number of firstorder radial modes exclusively, compared to standard SCFs in which, for example, excitation of the $\mathrm{EH}_{1,1}, \mathrm{HE}_{3,1}$ and $\mathrm{EH}_{2,1}$ modes inevitably occurs concurrently with that of the (unwanted) $\mathrm{HE}_{1,2}$ second-order radial mode.

In a more general way, the $m$ th radial order condition is determined by the cutoff of the $\mathrm{HE}_{1, m}$ mode. This condition is simply given by

$$
V_{0, \text { cutoff }}=\frac{(m-1) \pi}{1-\rho}+\epsilon
$$

where $\epsilon$, as well as the derivation of this equation, is given in Appendix E. In Fig. 6, we plot the boundaries that delimit the first radial order regions. For the important and practical case of $m=2$, the cutoff condition (28) can be further simplified to $V_{0, \text { cutoff }} \approx \frac{\pi}{1-\rho}$, which explicitly defines the upper boundary of the single radial order condition. In most cases, the correction term $\epsilon$ can be neglected without sacrificing much accuracy. This formula is no longer precise when $\rho$ approaches zero. However, as $\rho \rightarrow 0$, the fiber approaches SCF, and we can simply use the roots of $J_{1}\left(V_{0}\right)$ to find the cutoffs. 
TABLE V

VALUES OF $n_{0}^{2}$ IN REPORTED ANNULAR FIBERS

\begin{tabular}{lcc}
\hline Paper & $n_{0}^{2}$ & Reference \\
\hline Yan2012 & 1.0008 & {$[15]$} \\
Doerr2011 & 1.0052 & {$[17]$} \\
Kasahara2014 & $1.016,1.006-1.020$ & {$[9]$} \\
Yue2012 & $1.014,1.042,1.070$ & {$[4]$} \\
Jin2013 & 1.020 & {$[8]$} \\
Ramachandran2009 & 1.032 & {$[3]$} \\
Li2013 & 1.13 & {$[16]$} \\
\hline
\end{tabular}

\section{E. OAM fibers and fiber designs in the literature}

In this section we take published parameters for OAM fibers and OAM fiber designs and plot them on a single modal map. Our goal is to put into perspective the similarities and differences of these fibers, to identify proximity of designs to the cutoff condition, and highlight the utility of an analytical solution for the RCF vector cutoff conditions.

Fig. 7 shows normalized parameters of different reported RCF. We generated this plot using the weakly guiding approximation, as in all reported cases $n_{0}^{2}$ is close enough to one to justify this assumption. As a reference, with give values of $n_{0}^{2}$ in Table $\mathrm{V}$. Including variable $n_{0}^{2}$ would need another dimension in the plot of the modal map. When $n_{2}$ was not explicitly specified, we assumed $n_{2}=1.444$. When only a specific fiber at a specific wavelength was reported, we indicated it with a star; when a range of parameters were tested, we plotted the range using a solid line. Red and blue regions behind indicate the number of scalar modes supported for given $V_{0}$ and $\rho$ parameters, the red region being the single radial order region. Results from Yue2012 [4], Yan2012 [15], Li2013 [16], and Kasahara2014 [9] (dashed rectangles on the graph) are simulations, while results from Ramachandran2009 [3], Doerr2011 [17], and Jin2013 [8] (solid rectangles) are experimental. The fiber in [3] is not exactly annular, but we approximated it to get a comparison point. According to our simulations, it should support the third group of modes $\left(\mathrm{HE}_{3,1}, \mathrm{EH}_{1,1}\right)$, but it does not support $\mathrm{OAM}_{ \pm 2,1}$, because effective indices of the two vector modes are too close. Fiber in [16] is multicore, but we only considered one core. In all cases, the number of modes we predict using our cutoff relations is in agreement with the reported number of modes. It is interesting to note that all the reported RCF designs to date, with the exception of [9], operate in the single radial order condition (red tinted region).

\section{CONCLUSION}

In this paper, we performed a vector mode analysis of annular core optical fibers (RCFs) and fully derived the cutoff conditions for all the vector modes. We then showed that those equations were in full agreement with the cutoff equations of LP modes obtained with the weakly guiding approximation. Furthermore, we gave the LP mode characteristic equation in a much simpler and compact form than what was previously reported. Analytical results were verified using numerical simulations.

We have shown that precise knowledge of the modal cutoff frequencies is critical to design a RCF tailored for guiding

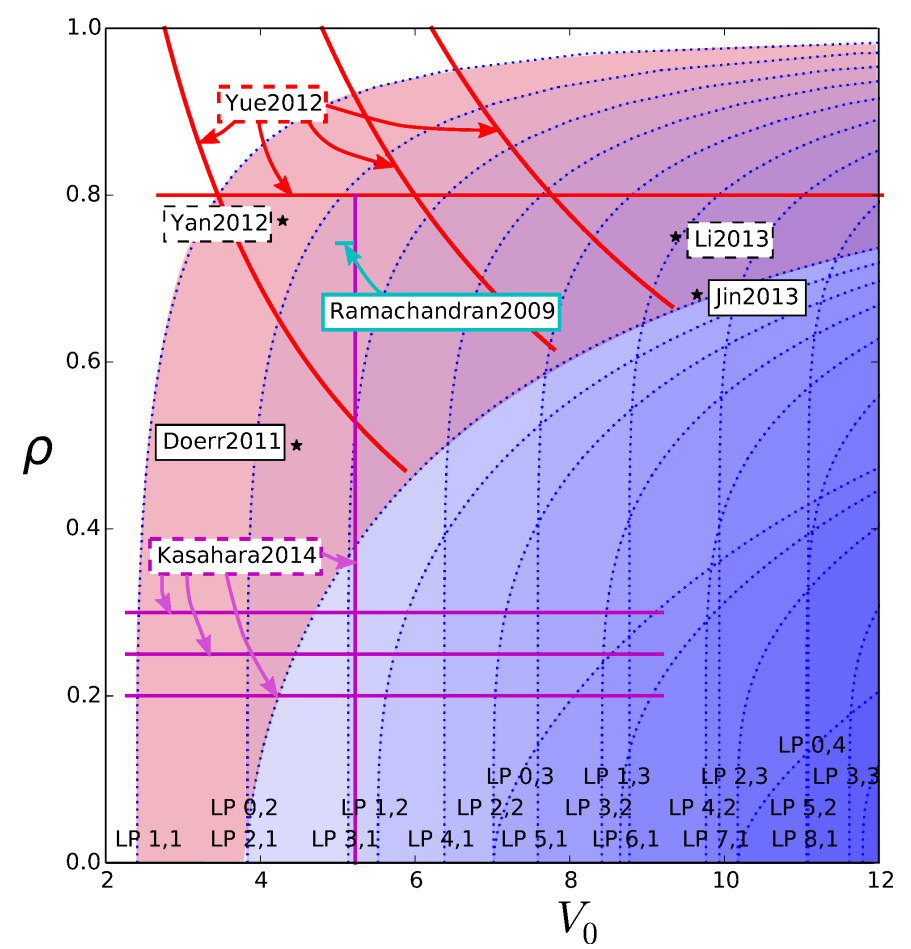

Fig. 7. Parameters of different reported annular fibers, compared to modal regions in weakly guiding approximation. Dashed rectangular boxes around author names indicate simulation results; solid rectangles indicate fibers that were produced and characterized.

cylindrical vector modes or for OAM-carrying fibers. Chiefly, it allows the fiber designer to know precisely the number of supported modes as function of the design parameters. Most notably, we provided a single radial order condition via a simple analytical expression which enables to identify the relevant wave guiding parameters permitting the exclusive guiding of vector modes or OAM modes presenting a single intensity ring that is well-matched to the studied RCF index profile.

\section{APPENDiX A}

SELECTEd BESSEL FUNCTION IDENTITIES

\section{A. Recurrence relations}

$$
\begin{gathered}
2 m Z_{m}(x)=x\left[Z_{m-1}(x)+Z_{m+1}(x)\right] \\
Z_{m}^{\prime}(x)=Z_{m-1}-\frac{m}{x} Z_{m}=-Z_{m+1}+\frac{m}{x} Z_{m}
\end{gathered}
$$

where $Z_{m}(x)$ is either $J_{m}(x)$ or $N_{m}(x)$.

\section{B. Asymptotic behavior when $x \rightarrow 0$}

$$
\begin{aligned}
\lim _{x \rightarrow 0} J_{m}(x) & =\left(\frac{x}{2}\right)^{m} m ! \\
\lim _{x \rightarrow 0} N_{m}(x) & = \begin{cases}\left(\frac{2}{\pi}\right) \ln x & m=0 \\
-\left(\frac{2}{x}\right)^{m} \frac{(m-1) !}{\pi} & m \geq 1\end{cases}
\end{aligned}
$$




$$
\begin{aligned}
\lim _{x \rightarrow 0} \frac{I_{m}^{\prime}(x)}{x I_{m}(x)} & = \begin{cases}\frac{1}{2} & m=0 \\
\frac{m}{x^{2}}+\frac{1}{2(m+1)} & m \geq 1\end{cases} \\
\lim _{x \rightarrow 0} \frac{K_{m}^{\prime}(x)}{x K_{m}(x)} & = \begin{cases}\frac{1}{x^{2} \ln x} & m=0 \\
-\frac{1}{x^{2}}-\ln x & m=1 \\
-\frac{m}{x^{2}}-\frac{1}{2(m-1)} & m>1\end{cases}
\end{aligned}
$$

C. Asymptotic behavior when $x \rightarrow \infty$

$$
\begin{aligned}
\lim _{x \rightarrow \infty} J_{\nu}(x) & =\sqrt{\frac{2}{\pi x}} \cos \varphi \\
\lim _{x \rightarrow \infty} N_{\nu}(x) & =\sqrt{\frac{2}{\pi x}} \sin \varphi
\end{aligned}
$$

where

$$
\varphi=x-\frac{\nu \pi}{2}-\frac{\pi}{4}
$$

\section{APPENDIX B \\ Details of THE CALCULATION OF THE CUTOFF FREQUENCIES}

Assuming that at $w^{2} \rightarrow 0$, (11) makes that (5) and (6) are zero. We are left to solve for the system of equations (7) and (8), where $A_{2}$ and $B_{2}$ are changed in term of $A_{1}$ and $B_{1}$ according to (11). Here it is convenient to introduce the function $\mathcal{F}_{\nu}(u b)$

$$
\mathcal{F}_{\nu}(u b)=J_{\nu}(u b)-\gamma_{a} N_{\nu}(u b)
$$

where

$$
\gamma_{a}=\frac{J_{\nu}(u a)}{N_{\nu}(u a)}
$$

$\mathcal{F}_{\nu}(u b)$ is a combination of the two Bessel functions $J_{\nu}(u b)$ and $N_{\nu}(u b)$, and thus satisfy all the recurrence relations of $Z_{m}$ of Appendix A.

Now, (7) and (8) can be written as

$$
\begin{aligned}
& A_{1}\left(\frac{1}{u^{2}}+\frac{1}{w^{2}}\right) \frac{\nu \beta}{b^{2}} \mathcal{F}_{\nu}(u b)= \\
& B_{1} k_{0}\left[\frac{\mathcal{F}_{\nu}^{\prime}(u b)}{u b}+\frac{1}{(w b)} \frac{K_{\nu}^{\prime}(w b)}{K_{\nu}(w b)} \mathcal{F}_{\nu}(u b)\right] \\
& B_{1}\left(\frac{1}{u^{2}}+\frac{1}{w^{2}}\right) \frac{\nu \beta}{b^{2}} \mathcal{F}_{\nu}(u b)= \\
& A_{1} k_{0}\left[\frac{n_{1}^{2}}{u b} \mathcal{F}_{\nu}^{\prime}(u b)+\frac{n_{2}^{2}}{(w b)} \frac{K_{\nu}^{\prime}(w b)}{K_{\nu}(w b)} \mathcal{F}_{\nu}(u b)\right]
\end{aligned}
$$

The determinant of this system of 2 equations

$$
\begin{aligned}
& \left(\frac{1}{u^{2}}+\frac{1}{w^{2}}\right)^{2} \frac{\nu^{2} \beta^{2}}{b^{4}}\left[\mathcal{F}_{\nu}(u b)\right]^{2}= \\
& k_{0}^{2}\left\{\frac{1}{u b} \mathcal{F}_{\nu}^{\prime}(u b)-\left[\frac{\nu}{(w b)^{2}}+\frac{1}{2(\nu-1)}\right] \mathcal{F}_{\nu}(u b)\right\} \times \\
& \left\{\frac{n_{1}^{2}}{u b} \mathcal{F}_{\nu}^{\prime}(u b)-n_{2}^{2}\left[\frac{\nu}{(w b)^{2}}+\frac{1}{2(\nu-1)}\right] \mathcal{F}_{\nu}(u b)\right\}
\end{aligned}
$$

where the asymptotic form of $\frac{K_{\nu}^{\prime}(w b)}{w b K_{\nu}(w b)}$ have been used for $\nu>1$.

We note that

$$
\left(\frac{1}{u^{2}}+\frac{1}{w^{2}}\right)^{2} \beta^{2}=\left[\frac{n_{1}^{2}}{u^{4}}+\frac{n_{1}^{2}+n_{2}^{2}}{u^{2} w^{2}}+\frac{n_{2}^{2}}{w^{4}}\right] k_{0}^{2}
$$

where $\beta^{2}$ have be written in term of $u^{2}$ and $w^{2}$ (see (4)).

Evaluating (B.41) for $w^{2} \rightarrow 0$, we obtain

$$
\begin{aligned}
\mathcal{F}_{\nu}(u b)\left\{\mathcal{F}_{\nu}(u b)-\right. & \left(n_{0}^{2}+1\right) \frac{(\nu-1)}{(u b)^{2}} \\
& {\left.\left[\nu \mathcal{F}_{\nu}(u b)+(u b) \mathcal{F}_{\nu}^{\prime}(u b)\right]\right\}=0 }
\end{aligned}
$$

for $\nu>1$.

Now, assuming that $\mathcal{F}_{\nu}(u b) \neq 0$ and using the recurrence relation for the Bessel functions, the cutoff frequencies are calculated from

$$
\mathcal{F}_{\nu-2}=\frac{\left(1-n_{0}^{2}\right)}{\left(1+n_{0}^{2}\right)} \mathcal{F}_{\nu}
$$

for $\nu>1$. After writing explicitly $\mathcal{F}_{\nu}(u b)$, the cutoff frequencies of the $\mathrm{HE}_{\nu, m}$ modes (12) is obtained.

For $\nu=1$, the asymptotic behavior of $\frac{K_{1}^{\prime}(w b)}{w b K_{1}(w b)}$ is logarithmic and in order to satisfy the relation (B.43), we have to conclude that $\mathcal{F}_{1}(u b)=0$, which gives the cutoff frequencies of the $\mathrm{HE}_{1, m}$ modes (13).

Notice also that $\mathcal{F}_{\nu}(u b)=0$ will satisfy equation (B.43), but will imply that at $r=b$, the axial electric and magnetic fields are zero. This is the case for a standard fiber, and also for $\mathrm{EH}_{\nu, m}$ modes in RCF.

We now consider the situation where we have

$$
\begin{aligned}
& A_{1} J_{\nu}(u b)+A_{2} N_{\nu}(u b)=0 \\
& B_{1} J_{\nu}(u b)+B_{2} N_{\nu}(u b)=0
\end{aligned}
$$

Introducing the function $\mathcal{G}_{\nu}(u a)$

$$
\mathcal{G}_{\nu}(u a)=J_{\nu}(u a)-\gamma_{b} N_{\nu}(u a)
$$

where

$$
\gamma_{b}=\frac{J_{\nu}(u b)}{N_{\nu}(u b)}
$$

Equations (7) and (8) are zero for $w^{2} \rightarrow 0$ and we will solve the system of equations (5) and (6), after writing $A_{2}$ and $B_{2}$ according to equation (B.45). Proceeding as before, we end up with

$$
\begin{aligned}
\mathcal{G}_{\nu}(u a)\left\{\mathcal{G}_{\nu}(u a)-\right. & \left(n_{0}^{2}+1\right) \frac{(\nu+1)}{(u a)^{2}} \\
& {\left.\left[\nu \mathcal{G}_{\nu}(u a)-(u a) \mathcal{G}_{\nu}^{\prime}(u a)\right]\right\}=0 }
\end{aligned}
$$

for $\nu \geq 1 . \mathcal{G}_{\nu}(u a) \neq 0$ in order to avoid finding a trivial solution, the cutoff frequencies of the $\mathrm{EH}_{\nu, m}$ modes can be calculated according to

$$
\mathcal{G}_{\nu+2}(u a)=\frac{\left(1-n_{0}^{2}\right)}{\left(1+n_{0}^{2}\right)} \mathcal{G}_{\nu}(u a)
$$

where the recurrence relation was used in (B.48). 


\section{APPENDIX C}

DERIVATION OF THE SCALAR CUTOFF EQUATIONS

Cutoff condition for scalar modes is given by (8) in [10], and in a very similar form in equation (13) of [11]. Using our notation, this equation is equivalent to:

$$
\begin{gathered}
{\left[1-\frac{\nu}{u a} \frac{J_{\nu}(u a)}{J_{\nu}^{\prime}(u a)}\right]\left[\frac{\nu}{u b} \frac{N_{\nu}(u b)}{N_{\nu}^{\prime}(u a)}+\frac{N_{\nu}^{\prime}(u b)}{N_{\nu}^{\prime}(u a)}\right]=} \\
{\left[1-\frac{\nu}{u a} \frac{N_{\nu}(u a)}{N_{\nu}^{\prime}(u a)}\right]\left[\frac{\nu}{u b} \frac{J_{\nu}(u b)}{J_{\nu}^{\prime}(u a)}+\frac{J_{\nu}^{\prime}(u b)}{J_{\nu}^{\prime}(u a)}\right]}
\end{gathered}
$$

While this seems rather complex, we will show it can be reduced to (20).

We first multiply both sides by $J_{\nu}^{\prime}(u a) N_{\nu}^{\prime}(u a)$ :

$$
\begin{array}{r}
{\left[J_{\nu}^{\prime}(u a)-\frac{\nu}{u a} J_{\nu}(u a)\right]\left[\frac{\nu}{u b} N_{\nu}(u b)+N_{\nu}^{\prime}(u b)\right]=} \\
\quad\left[N_{\nu}^{\prime}(u a)-\frac{\nu}{u a} N_{\nu}(u a)\right]\left[\frac{\nu}{u b} J_{\nu}(u b)+J_{\nu}^{\prime}(u b)\right]
\end{array}
$$

Then we use (A.30) to transform all the derivatives:

$$
\begin{gathered}
{\left[\frac{\nu}{u a} J_{\nu}(u a)-J_{\nu+1}(u a)-\frac{\nu}{u a} J_{\nu}(u a)\right]} \\
{\left[\frac{\nu}{u b} N_{\nu}(u b)+N_{\nu-1}(u b)-\frac{\nu}{u b} N_{\nu}(u b)\right]=} \\
{\left[\frac{\nu}{u a} N_{\nu}(u a)-N_{\nu+1}(u a)-\frac{\nu}{u a} N_{\nu}(u a)\right]} \\
{\left[\frac{\nu}{u b} J_{\nu}(u b)+J_{\nu-1}(u b)-\frac{\nu}{u b} J_{\nu}(u b)\right]}
\end{gathered}
$$

This simplifies to:

$$
J_{\nu+1}(u a) N_{\nu-1}(u b)=N_{\nu+1}(u a) J_{\nu-1}(u b)
$$

\section{APPENDIX D}

\section{RELATION BETWEEN CUTOFF SEPARATION AND EFFECTIVE} INDEX SEPARATION

As stated in section IV-C, a rough idea of the separation between the effective indices of the modes can be found from the modal map. Effective index separation between modes is often a design criteria for RCF, especially for OAM mode propagation. Effective index separation correlates with normalized frequency $\left(V_{0}\right)$ separation between modes. Modal maps can serve as a first order approximation of normalized frequency separation, and hence effective index separation. Calculation of $V_{0}$ at cutoff is computationally fast using equations provided in table IV. These equations can be used as the first step in the design process, restricting fiber parameters (to be searched via computationally heavy simulations) to a region with promising prospects for the desired effective index separation.

Consider a modal map such as that in Fig. 4 or Fig. 5, with a horizontal line fixing a value for the $\rho$ parameter. The distance, in terms of $V_{0}$, between the boundaries of two different modes intersecting this horizontal line is what we call the cutoff separation. Consider next the curve that gives normalized propagation constants as a function of $V_{0}$, such as Fig. 2 or Fig. 8. In these plots, the cutoff separation corresponds to the horizontal distance between the two mode curves at $\widetilde{\beta}=0$, i.e., along the abscissa. We are interested in the separation in terms of effective indices (or propagation constants) between

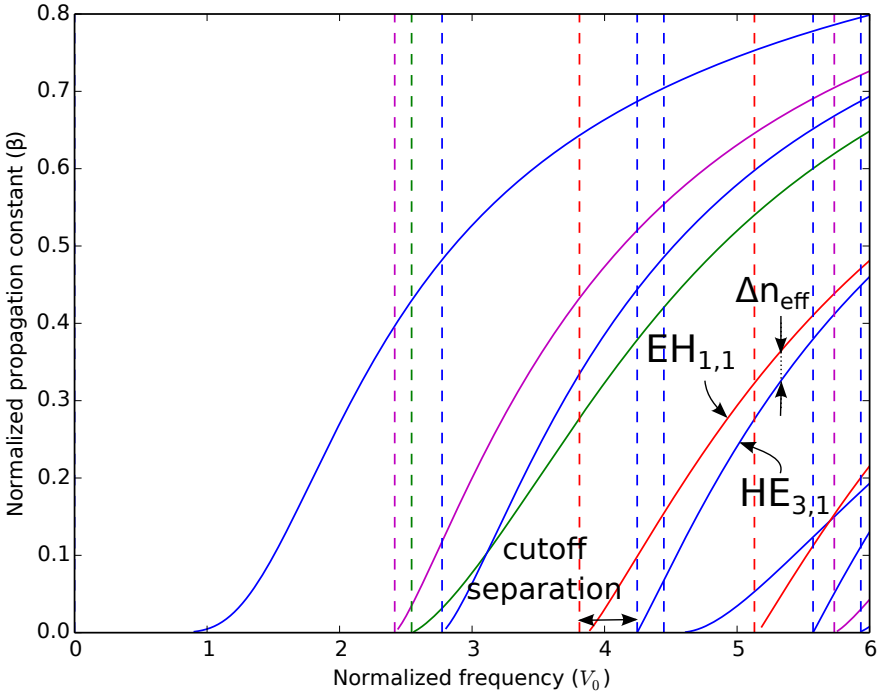

Fig. 8. Normalized propagation constant $\widetilde{\beta}$ as a function of the normalized frequency $V_{0}$, for RCF with $\rho=0.25$ and $n_{0}^{2}=2.085$.

two modes, for a given wavelength (i.e., a given value of $V_{0}$ ). We read this on Fig. 2 and Fig. 8 as the vertical distance between two lines for a given $V_{0}$. Assuming that the curves between two modes inside a given mode group $\left(\mathrm{HE}_{\nu+1, m^{-}}\right.$ $\mathrm{EH}_{\nu-1, m}$ ) are roughly following each other, we can infer that the greater the separation between cutoffs inside a mode group, the greater the separation between their effective indices. This is purely a qualitative inference that can be used as a rule of thumb to find promising fiber parameters. These parameters must be probed via numerical simulations of a fiber design to verify the targeted performance.

\section{APPENDIX E}

ASYMPTOTIC EXPANSION FOR THE CUTOFF OF $\mathrm{HE}_{1, m}$

In [18], equations (9.5.27)-(9.5.29) give an asymptotic expansion of the $s$ th zero of the cross-product function

$$
J_{\nu}(z) N_{\nu}(\lambda z)-J_{\nu}(\lambda z) N_{\nu}(z)
$$

Using $z=\rho V_{0}, \lambda=1 / \rho, \nu=1$, and $s=m-1$, we can directly use this development to solve for the cutoff conditions of the $\mathrm{HE}_{1, m}$ modes. The first term of the expansion becomes:

$$
V_{0}=\frac{z}{\rho} \approx \frac{\gamma}{\rho}=\frac{s \pi}{\rho(\lambda-1)}=\frac{(m-1) \pi}{1-\rho}
$$

On a similar way, we can develop the other terms of the expansion as

$$
\epsilon=\frac{p}{\rho \gamma}+\frac{q-p^{2}}{\rho \gamma^{3}}+\frac{r-4 p q+2 p^{2}}{\rho \gamma^{5}}+\cdots
$$

where

$$
\begin{aligned}
\gamma & =\frac{(m-1) \pi \rho}{1-\rho} & p & =\frac{3}{8} \rho \\
q & =-\frac{21}{128} \frac{\left(1-\rho^{3}\right)}{(1-\rho)} \rho & r & =\frac{1899}{5120} \frac{\left(1-\rho^{5}\right)}{(1-\rho)} \rho
\end{aligned}
$$

However, we must be very careful when we use this, because it becomes numerically unstable when $\rho$ is small. 


\section{REFERENCES}

[1] D. J. Richardson, J. M. Fini, and L. E. Nelson, "Space-division multiplexing in optical fibres," Nat Photon, vol. 7, no. 5, pp. 354-362, May 2013.

[2] P. Solarik, Z. Burian, I. Kasik, V. Matejec, J. Mrazek, and M. Hayer, "Dielectric annular core fiber for optical sensing," Proc. SPIE, vol. 6189, pp. 61 891R-61 891R-10, 2006.

[3] S. Ramachandran, P. Kristensen, and M. F. Yan, "Generation and propagation of radially polarized beams in optical fibers," Opt. Lett., vol. 34, no. 16, pp. 2525-2527, Aug. 2009.

[4] Y. Yue, Y. Yan, N. Ahmed, J.-Y. Yang, L. Zhang, Y. Ren, H. Huang, K. Birnbaum, B. Erkmen, S. Dolinar, M. Tur, and A. Willner, "Mode properties and propagation effects of optical orbital angular momentum (oam) modes in a ring fiber," Photonics Journal, IEEE, vol. 4, no. 2, pp. 535-543, Apr. 2012.

[5] P. Gregg, P. Kristensen, S. Golowich, J. Olsen, P. Steinvurzel, and S. Ramachandran, "Stable transmission of 12 oam states in air-core fiber," in CLEO: 2013. OSA, 2013, p. CTu2K.2.

[6] C. Brunet, B. Ung, Y. Messaddeq, S. LaRochelle, E. Bernier, and L. Rusch, "Design of an optical fiber supporting 16 oam modes," in Optical Fiber Communication Conference. OSA, 2014, p. Th2A.24.

[7] N. Fontaine, R. Ryf, M. Hirano, and T. Sasaki, "Experimental investigation of crosstalk accumulation in a ring-core fiber," in Photonics Society Summer Topical Meeting Series, 2013 IEEE, July 2013, pp. 111-112.

[8] X. Jin, R. Li, D. O'Brien, and F. Payne, "Linearly polarized mode division multiplexed transmission over ring-index multimode fibres," in Photonics Society Summer Topical Meeting Series, 2013 IEEE, July 2013, pp. 113-114.

[9] M. Kasahara, K. Saitoh, T. Sakamoto, N. Hanzawa, T. Matsui, K. Tsujikawa, and F. Yamamoto, "Design of three-spatial-mode ring-core fiber,' J. Lightwave Technol., vol. 32, no. 7, pp. 1337-1343, Apr 2014.

[10] B. C. Sarkar, P. K. Choudhury, and T. Yoshino, "On the analysis of a weakly guiding doubly clad dielectric optical fiber with an annular core," Microw. Opt. Techn. Let., vol. 31, no. 6, pp. 435-439, 2001.

[11] J. Marcou and S. Février, "Comments on 'On the analysis of a weakly guiding doubly clad dielectric optical fiber with an annular core'," Microw. Opt. Techn. Let., vol. 38, no. 3, pp. 249-254, 2003.

[12] M. Hautakorpi and M. Kaivola, "Modal analysis of m-type-dielectricprofile optical fibers in the weakly guiding approximation," J. Opt. Soc. Am. A, vol. 22, no. 6, pp. 1163-1169, Jun 2005.

[13] A. W. Snyder and J. D. Love, Optical waveguide theory. Chapman and Hall, 1983.

[14] P. Yeh, A. Yariv, and E. Marom, "Theory of bragg fiber," J. Opt. Soc. Am., vol. 68, no. 9, pp. 1196-1201, Sep 1978.

[15] H. Yan, E. Zhang, B. Zhao, and K. Duan, "Free-space propagation of guided optical vortices excited in an annular core fiber," Opt. Express, vol. 20, no. 16, pp. 17904-17915, Jul 2012.

[16] S. Li and J. Wang, "A multi-ring multi-oam-mode fiber for high-density space-division multiplexing (7 rings x 22 oam modes)," in Photonics Conference (IPC), 2013 IEEE, Sept 2013, pp. 301-302.

[17] C. R. Doerr, N. Fontaine, M. Hirano, T. Sasaki, L. Buhl, and P. Winzer, "Silicon photonic integrated circuit for coupling to a ring-core multimode fiber for space-division multiplexing," in 37th ECOC Conference. OSA, 2011, p. Th.13.A.3.

[18] M. Abramowitz and I. A. Stegun, Handbook of mathematical functions with formulas, graphs, and mathematical tables, ser. National Bureau of Standards Applied Mathematics Series. For sale by the Superintendent of Documents, U.S. Government Printing Office, Washington, D.C., 1964, vol. 55.

Bora Ung received from the Université Laval in Quebec City the B.Eng degree in engineering physics (2005) and the M.Sc. in physics (2007, Honours) during which he studied surface plasmons diffraction in nanoscale structures. $\mathrm{He}$ obtained the Ph.D. degree in engineering physics (2012, with distinction) from the Ecole Polytechnique de Montréal where he worked on novel microstructured optical fibers for mid-infrared and terahertz waveguiding. He was a postdoctoral fellow at the Center for optics, photonics and lasers (COPL) developing specialty few-mode fibers for space-division multiplexing. He joined the Ecole de Technologie Supérieure as an assistant professor in the fall of 2014. His research interests include fiber optic transmission of vector modes and modes carrying orbital angular momentum, fiber sensors, plasmonics, terahertz waves and non-reciprocal devices.
Charles Brunet graduated in computer engineering in 2010, and received M.Sc. degree in electrical engineering in 2012, at Université Laval, Canada. $\mathrm{He}$ is currently working toward the Ph.D. in the Department of Electrical and Computer Engineering, Center for Optics, Photonics and Lasers (COPL) of Université Laval. His research interests include optical fiber design and numerical simulation for the use of OAM in optical communication systems.

Pierre-André Bélanger is now professor emeritus at Université Laval and adjunct professor at the COPL center. His actual research interest concentrates around basic electromagnetism problems such as optical fiber devices and beams propagation. He is a fellow of OSA and live member of IEEE and OSA.

Younès Messaddeq who holds a PhD in solid state chemistry (1990) and a bachelors degree in solid state chemistry from the Universitéde Rennes 1, France, is one of the worlds most accomplished researchers in materials development for optics and photonics. In his academic research, he has published over 331 papers in refereed journals and conferences, and presented several invited talks. His $\mathrm{H}$-index is 35 , and has well over 4600 citations of his published works. In 2010, he was appointed to one of the 19 prestigious Canada Excellence Research Chairs (CERC), providing 28M\$ in research funds at UniversitéLaval, Quéec, Canada. He has been a visiting professor at the Physics Institute of So Carlos, Brazil; the Universitéde Bourgogne, France; and the University of Münster, Germany. He has also served as a visiting researcher and fellow at the National Institute for Inorganic Materials in Tsukuba, Japan. He has reported on his work in high-impact international publications.

Sophie Larochelle (M'00) received a Bachelor's degree in engineering physics from Université Laval, Canada, in 1987; and a Ph.D. degree in optics from the University of Arizona, USA, in 1992. From 1992 to 1996, she was a Research Scientist at the Defense Research and Development Canada - Valcartier, where she worked on electro-optical systems. She is now a professor at the Department of Electrical and Computer Engineering, Université Laval, where she holds a Canada Research Chair (Tier 1) in Advanced Photonics Technologies for Emerging Communication Strategies. Her current research activities are focused on active and passive components for optical communication systems including Bragg gratings filters, optical amplifiers, silicon photonic devices, multi- wavelength and pulsed lasers. Other research interests include spatial division multiplexing, all-optical signal processing and routing, and transmission of radio-over-fiber signals including UWB and GPS. Dr. LaRochelle is a senior member of OSA and IEEE.

Leslie Ann Rusch (S'91-M'94-SM'00-F'10) received the B.S.E.E. degree (with honors) from the California Institute of Technology, Pasadena, in 1980 and the M.A. and Ph.D. degrees in electrical engineering from Princeton University, Princeton, NJ, in 1992 and 1994, respectively. Dr. Rusch has experience in defense, industrial and academic communications research. She was a communications project engineer for the Department of Defense from 1980-1990. While on leave from Université Laval, she spent two years (2001-2002) at Intel Corporation creating and managing a group researching new wireless technologies. She is currently a Professor in the Department of Electrical and Computer Engineering at Université Laval, QC, Canada, performing research on wireless and optical communications. Prof. Rusch's research interests include digital signal processing for coherent detection in optical communications, spatial multiplexing using orbital angular momentum modes in fiber, radio over fiber and OFDM for passive optical networks; and in wireless communications, optimization of the optical/wireless interface in emerging cloud based computing networks, optical pulse shaping for high-bit rate ultrawide-band (UWB) systems, and implantable medical sensors with high bit rate UWB telemetry. She is recipient of the IEEE Canada J. M. Ham Award for Graduate Supervision. Prof. Rusch has published over 100 journal articles in international journals (90\% IEEE/IEE) with wide readership, and contributed to over 130 conferences. Her articles have been cited over 3600 times per Google Scholar. 